\title{
EmoRemSys: Sistema de recomendación de recursos educativos basado en detección de emociones
}

\author{
Maritza Bustos López ${ }^{1}$, Agustín Job Hernández Montes ${ }^{1}$ Raquel Vásquez Ramírez , \\ Giner Alor Hernández ${ }^{1}$, Ramón Zatarain Cabada², María Lucia Barrón Estrada² \\ maritbustos@gmail.com, agus_job@hotmail.com,omeg_22@hotmail.com, galor@itorizaba. \\ edu.mx, rzatarain@itculiacan.edu.mx, mbarron@itculiacan.edu.mx \\ ${ }^{1}$ División de Estudios de Postgrado e Investigación, Instituto Tecnológico de Orizaba, Avenida Oriente 9 No. \\ 852 Col. Emiliano Zapata, 94330, Orizaba, Veracruz, Orizaba, México. \\ ${ }^{2}$ División de Estudios de Postgrado e Investigación, Instituto Tecnológico de Culiacán. Av. Juan de Dios \\ Bátiz 310 Pte. Col. Guadalupe. C. P. 80220. Culiacán, México.
}

DOI: $10.17013 /$ risti.17.80-95

Resumen: Actualmente existen diversos repositorios de recursos educativos los cuales brindan apoyo a los estudiantes en el proceso de enseñanza-aprendizaje. No obstante uno de los problemas que se les presentan a los estudiantes al hacer búsquedas específicas de recursos en diferentes repositorios es que se encuentran con un gran número de resultados y en ocasiones pierden demasiado tiempo para seleccionar un recurso o no encuentran lo que necesitan. En este trabajo se presenta EmoRemSys un sistema de recomendación de recursos educativos basado en técnicas de computación afectiva que permite localizar recursos educativos con base en un análisis sentimental del estudiante haciéndolo adaptable a las necesidades del usuario. Se describe a detalle el proceso de recomendación basado en filtrado colaborativo y el proceso de obtención del sentimiento. Los resultados muestran un alto porcentaje de precisión en las recomendaciones hechas por EmoRemSys.

Palabras-clave: Análisis de Sentimientos, Computación Afectiva, Sistemas de Recomendación.

\section{EmoRemSys: An educational recommender system by using emotions detection}

Abstract: Nowadays, there are several repositories of educational resources which
provide support to students in the teaching-learning process. However one of the
problems that are presented to students to make specific searches for resources in
different repositories is found with a large number of results and sometimes lose too
much time to select a resource or can not find what they need. This paper presents
EmoRemSys an educational recommender system based on affective computing
techniques for locating educational resources by using emotions detection. The
recommendation and emotion detection processes are described in detail. Results
showahigh percentage of precision about recommendations provided by EmoRemSys.

Keywords: Sentiment Analysis, Affective Computing, Recommender Systems. 


\section{Introducción}

El crecimiento exponencial de información en la Web dificulta la exploración e identificación de recursos que satisfagan las necesidades de un usuario, en los últimos años han surgido repositorios de recursos educativos como apoyo a los estudiantes con deficiencias en conocimiento (Aguila, 2010). Desafortunadamente en ocasiones es necesario buscar en diversos repositorios ya que uno solo no es suficiente para encontrar los recursos que el estudiante necesite, este puede tomar demasiado tiempo y no alcanzar el objetivo deseado el cual es encontrar diversos recursos que deben cubrir las demandas de una materia o tema en específico que el estudiante necesite. Los sistemas de recomendación son herramientas que generan sugerencias sobre un determinado objeto de estudio, a partir de las preferencias y opiniones dadas por otros usuarios (Beltrán Páez Germán, 2015). El filtrado colaborativo basa sus recomendaciones sobre las calificaciones o el comportamiento de otros usuarios en el sistema, esta técnica asume que si a un grupo de usuarios les gustan las mismas cosas que el usuario activo "X" por lo tanto al usuario "X" es probable que le gusten las cosas que todavía no ha visto de aquellos usuarios (Michael D. Ekstrand, 2015). Los sistemas de recomendación han tenido un fuerte impacto en diferentes áreas como son medicina, entretenimiento, comercio electrónico, educativa entre otras (Betancur, 2009) (Castillejo, 2007).

Los sentimientos son el resultado de las emociones y significan un estado de ánimo afectivo que se presenta en una persona, las emociones son expresiones psicofisiológicas, bilógicas y de estados mentales, también se pueden definir como adaptaciones del individuo a estímulos provocados por el entorno, se ha demostrado que las emociones afectan en la mayoría de las actividades humanas entre las cuales están la creatividad, la toma de decisiones y la comunicación (Barrón-Estrada, 2014). En lo que respecta al análisis de sentimientos, se hace uso de la computación afectiva la cual es de suma importancia en el desarrollo de EmoRemSys permitiendo que el estudiante se sienta más cómodo adaptando las recomendaciones a su estado de ánimo. La Computación Afectiva (Affective Computing) es una disciplina de la Inteligencia Artificial que intenta desarrollar métodos computacionales orientados a reconocer emociones humanas y generar emociones sintéticas (Causa, 2007). Para la solución a estos problemas se presenta EmoRemSys, la importancia que tiene el desarrollo de este sistema es brindar al estudiante un apoyo para la búsqueda de recursos educativos de diversos repositorios permitiendo ahorrarse tiempo y además haciendo dichas recomendaciones a partir de su estado de ánimo mediante un análisis de sentimientos.

Estedocumento está estructuradodelasiguientemanera, enla Sección 2 sepresentael estado del arte referente a los diversos trabajos relacionados con los sistemas de recomendación y computación afectiva. En la sección 3 se describe la arquitectura del sistema EmoRemSys. En la sección 4 se presentan el proceso de generación de recomendaciones y el proceso de obtención del sentimiento. En la Sección 5 se presenta un caso de estudio generación de recomendaciones de recursos educativos. En la Sección 6 se presentan las evaluaciones, finalmente en la Sección 7 se presentan las conclusiones y trabajo a futuro.

\section{Estado del Arte}

A continuación se presenta una revisión del estado del arte sobre los trabajos relacionados con los sistemas de recomendación y computación afectiva. 


\subsection{Sistemas de recomendación aplicaciones y enfoques.}

En (Bustos-López et. al., 2015) se presentó una arquitectura genérica para el desarrollo de los sistemas de recomendación educativas independientes del tipo de recomendación generada. Asimismo, se identifican las características principales de un sistema de recomendación educativo. Estas características son componentes importantes para lograr los objetivos que tienen los sistemas de recomendación educativos con el fin de proporcionar información precisa a los estudiantes de acuerdo a sus preferencias, perfil de usuario y objetivos de aprendizaje. En (Kaklauskas et al., 2013) se presentó el desarrollo de un sistema de recomendación para analizar el rendimiento académico del estudiante. El sistema de recomendación determina el nivel de aprendizaje de la productividad integral empleando tres técnicas principales (fisiológica, psicológica y conductual). En (Zhang, Wang, \& Vassileva, 2013) se propuso un sistema centrado en el usuario llamado "SocConnect" en el que se da solución a dos problemas que enfrentan los usuarios de redes sociales: 1 ) dificultad para realizar un seguimiento de sus amistades sociales y las actividades sociales de amigos esparcidos por diferentes redes sociales, y (2) la gran cantidad de datos sociales que necesitan actualizarse (actualizaciones de los amigos y otras actividades). En (Hsu, 2013) se propuso un enfoque de aprendizaje móvil de idiomas basado en recomendaciones personalizadas. El sistema de aprendizaje móvil proporcionaba un mecanismo de recomendación de material EFL (Inglés como Lengua Extranjera) de lectura para guiar a los estudiantes a leer los artículos que se ajusten a sus preferencias y niveles de conocimiento teniendo de referencia los gustos de los demás estudiantes. En (Chia-Cheng, Hsin-Chin, Kuo-Kuang, \& Yueh-Min, 2012) se propuso un sistema de recomendación personalizado basado en el grado de dificultad de los materiales auxiliares, estilos individuales de aprendizaje y temas específicos de un curso. En (Pera, 2011) se desarrolló PBRecS, un sistema de recomendación de libros basado en las interacciones sociales e intereses personales para sugerir libros a los usuarios. PBRecS está basado en las amistades establecidas en LibraryThing, para generar sugerencias más personalizadas dependiendo de las amistades del usuario. En (Yang, 2014) (Nieto, 2007) se presentaron estudios y una descripción acerca de las técnicas y tipos de filtrado colaborativo. Adicionalmente se presenta también un análisis de los algoritmos y métricas ocupadas en el filtrado colaborativo que permiten el desarrollo de sistemas de recomendación. En (Poy \& Gonzales-Aguilar, 2014) se presentó un estudio y análisis de los MOOC (Cursos online abiertos y masivos), una de las herramientas que ha surgido de la combinación de e-learning y modelos de redes sociales, la cual se ha integrado en muchos programas educativos transformando las organizaciones e-learning a nivel mundial. A partir de los estudios previos, se identificaron en el análisis cuatro factores críticos que son (1)el diseño de software educativo, (2)las tasas de abandono, (3)el alcance de la universalización, y (4)la estrategia de negocio subyacente a esta oferta educativa. En (Moreno-Cadavid \& Montoya-Gómez, 2015) se desarrolló una herramienta virtual con contenido en el área de cálculo con una característica particular: la ludificación como estrategia didáctica. En esta herramienta se presentó una validación de dicha propuesta mediante un curso de pre-cálculo en la Universidad Nacional de Colombia.

\subsection{Implementación de técnicas de computación afectiva}

En (Liu, 2013) se presentó un estudio realizado a la importancia que tiene la computación afectiva en la educación a distancia y se presentaron algunos estudios realizados al 
aprendizaje de los estudiantes por medio de la obtención de emociones mediante el reconocimiento facial, fisiológico y auditivo lo cual permitía ajustar las clases y maestros observando los resultados de sus emociones adaptando clases a los gustos del estudiante. En (Tzu-Wei Tsai, 2012) se desarrolló un juego con varios niveles de dificultad que utiliza material de aprendizaje adaptativo, los niveles del juego están desarrollados para adaptarse a la habilidad de cada estudiante haciendo uso de emociones faciales. El juego identifica a través de los gestos del usuario el nivel de dificultad para adaptarlo a cada estudiante. En (Barrón-Estrada, 2014) (Yasmín Hernández, 2013) se presentaron sistemas tutores inteligentes STI para el aprendizaje de números naturales de zer grado y capacitación para operadores de sistemas eléctricos, los STI eran configurados por expertos en la materia para lograr una enseñanza optima a los estudiantes y operadores, permitiendo así mediante técnicas de computación afectiva adaptarse al entorno a través de la detección de gestos y así determinar si el usuario estaba comprendiendo, los STI se adaptan a lo que los usuarios necesitan para que la comprensión de los diferentes temas que se presentaban fuera más sencilla. En (Eirinaki, Pisal, \& Singh, 2011) se presenta un algoritmo el cual no solo analiza el sentimiento en general de un documento o reseña, también identifica la orientación semántica de componentes específicos de opinión que da lugar a un sentimiento particular. En (Wang, Zhu, \& Li, 2012) se presentó un sistema de revisión de síntesis en la Web "SumView", el cual permite extraer automáticamente las expresiones y opiniones de los clientes en la revisión de las características de diferentes productos.

Mediante estas investigaciones se obtuvo una idea más clara de cómo trabajan los sistemas de recomendación con el filtrado colaborativo y la computación afectiva en el ámbito educativo y se determinó que hasta el momento no hay una herramienta que integre estas dos tecnologías, con estas tecnologías se logran recomendaciones más precisas y adaptables a las necesidades del usuario mediante la detección de sentimientos.

\section{EmoRemSys: Arquitectura}

EmoRemSys (Emotional Recommender System) es un sistema de recomendación basado en filtrado colaborativo y computación afectiva. El objetivo de este sistema es realizar recomendaciones de recursos educativos permitiendo al usuario ahorrarse tiempo en la búsqueda de material que sea de ayuda para cubrir sus necesidades.

\subsection{Descripción de la Arquitectura}

La arquitectura de EmoRemSys está diseñada en 3 capas para organizar los componentes y distribuir las tareas y funcionalidades, los componentes están desarrollados a partir de las necesidades que se identificaron a lo largo del desarrollo del proyecto. La arquitectura se muestra en la figura 1. Cada capa contiene componentes que tienen funciones específicas las cuales se explican a continuación:

Capa de presentación: Esta capa presenta un conjunto de interfaces con las que cuenta EmoRemSys la cuales se desarrollaron en HTML5, CSS3, JavaScript. Esta capa es la que permite al usuario interactuar con el sistema permitiendo hacer búsqueda de los recursos educativos y presentando la información organizada.

Capa de servicios: Esta capa está formada por 2 módulos, el primer módulo llamado análisis sentimental contiene la API de SkyBiometry que permite obtener datos sobre 
la imagen de un rostro, dejando saber al analizador de imágenes el estado de ánimo del estudiante, los datos de cada foto tomada se envían al procesador de resultados donde se obtiene un promedio de los sentimientos analizados. El segundo módulo llamado recomendaciones es donde se encuentra la API de Apache Mahout que se ocupa de procesar los datos obtenidos por el módulo de análisis sentimental y aplicar los algoritmos necesarios para hacer la recomendaciones. Este módulo esta encapsulado en un servicio Web en Java el cual se invoca mediante el protocolo SOAP desde un sistema Web desarrollado en PHP para realizar dichas operaciones y obtener un resultado, el analizador de peticiones es el encargado de dirigir al módulo correspondiente dependiendo si el usuario tiene Webcam y se tomaron las fotos, se envían al módulo de sentimientos o en caso contrario al módulo de recomendaciones.

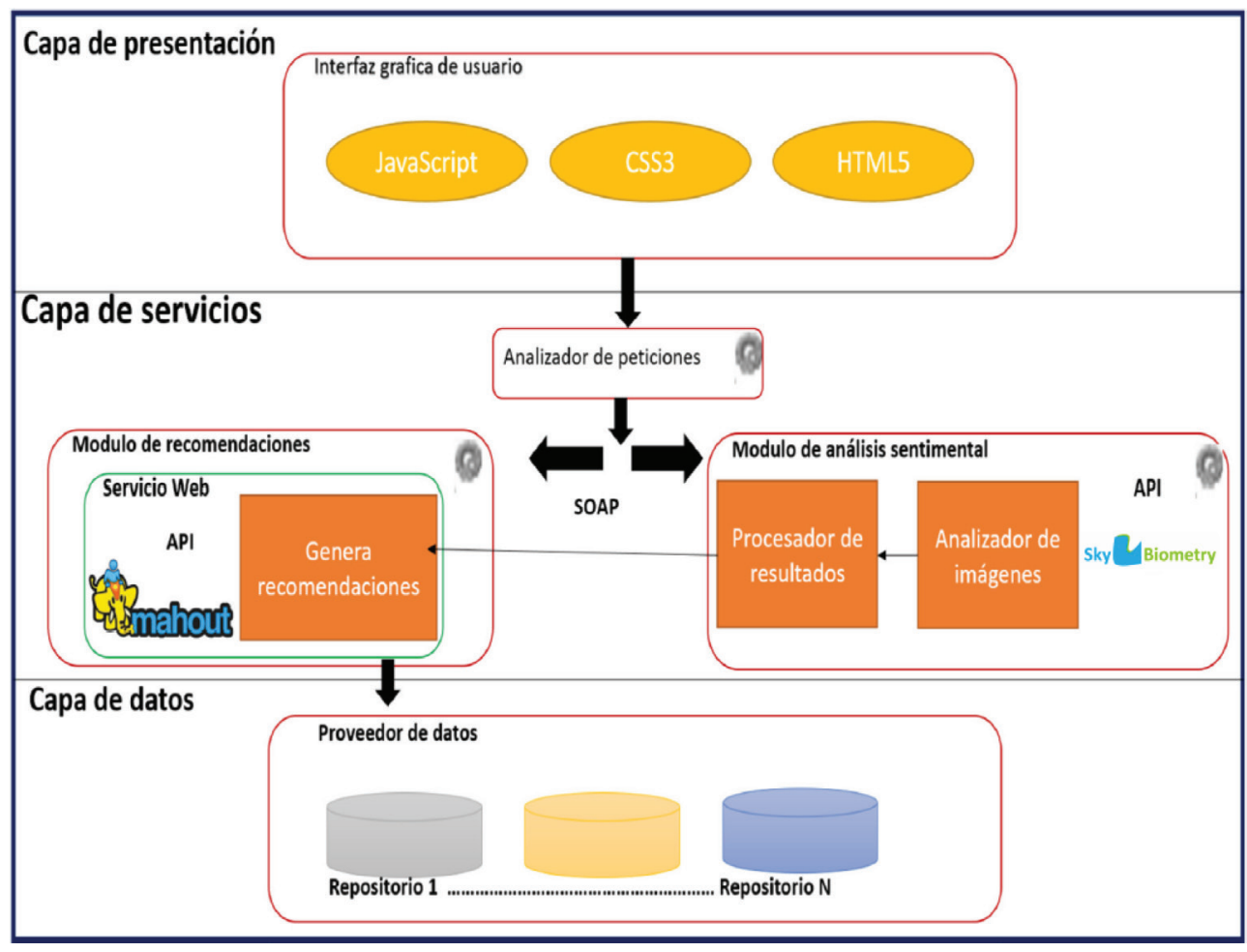

Figura 1 - Arquitectura de tres capas sistema EmoRemSys

Capa de datos: Esta capa contiene los diversos repositorios en donde están contenidos los recursos educativos con los cuales trabaja EmoRemSys.

A continuación se presenta una breve descripción de cada uno de los componentes que conforman la arquitectura de repositorios en donde están contenidos los recursos educativos con los cuales trabaja EmoRemSys.

Analizador de peticiones: Este componente es el encargado de capturar el resultado del usuario en cuanto a permitir el uso de su cámara Web para que le sean tomadas 
fotografías o la declinación del usuario a dicha petición. Una vez que obtiene el resultado este redirigirá a la página que muestra el recurso educativo con o sin uso de la cámara dependiendo de la decisión del estudiante.

Analizador de imágenes: El analizador de imágenes es un componente el cuál hace uso de la API de SkyBiometry haciendo una petición, dicha petición está constituida con la API key y API secret necesarias para hacer las llamadas a la API de SkyBiometry enviando la URL en donde se encuentra alojada la fotografía del usuario en el servidor. Una vez hecha la llamada la API de SkyBiometry devuelve el resultado de la información en formato JSON (JavaScript Object Notation).

Procesador de resultados: La operación que efectúa este componente es tratar la información recibida en formato JSON del Analizador de imágenes, decodificando la información y obteniendo la información necesario por partes en lo que se refiere al sentimiento del estudiante, procesa el resultado y envía el sentimiento o en caso de que la imagen no contenga un rostro envía el mensaje de la falta de sentimiento. Una vez realizado lo anterior descrito mediante el protocolo SOAP se hace una invocación al servicio Web desarrollado en donde se encuentra encapsulado el componente de recomendaciones, dicho servicio Web está desarrollado en Java por el uso de la API de Apache Mahout a este módulo se envían los datos recolectados del usuario como son su Id, el Id del recuso educativo y la calificación que el estudiante dio al recurso.

Genera recomendaciones: Este componente esta encapsulado en un servicio Web, dicho componente contiene la API de Apache Mahout la cual realiza las recomendaciones del filtrado colaborativo mediante los siguientes pasos:

1. Insertar en el conjunto de datos llamado EmoRemSys.csv el id del estudiante, el id del recurso y la calificación que el estudiante dio al recurso.

2. Calcular la similitud entre usuarios, esto se hace ocupando una métrica de similitud, la API de Apache Mahout cuenta con: Tanimoto coefficient similarity, Pearson correlation similarity, uncentered cosine similarity, Euclidean distance similarity, con estas métricas se calcula la similitud entre parejas de usuarios, la métrica ocupada por el sistema es coefficient similarity Pearson.

3. Una vez calculada la similitud entre usuarios Apache Mahout presenta una serie de algoritmos para calcular los vecinos más cercanos del usuario activo los cuáles son: NearestN User Neighborhood, Threshold User Neighborhood, Caching User Neighborhood en el cual se ocupó el algoritmo de NearestN User Neighborhood del cual se insertó un umbral para evitar que dichos recursos con un umbral menor sean recomendados.

4. Finalmente, se determinan las recomendaciones y se eligen los $\mathrm{N}$ recursos más adecuados para ser recomendados al usuario.

\section{Proceso de Generación de Recomendaciones y Proceso de Obtención del Sentimiento}

En la Figura 2 se presenta el proceso de generación de recomendaciones y se explican cada uno de los pasos involucrados. 


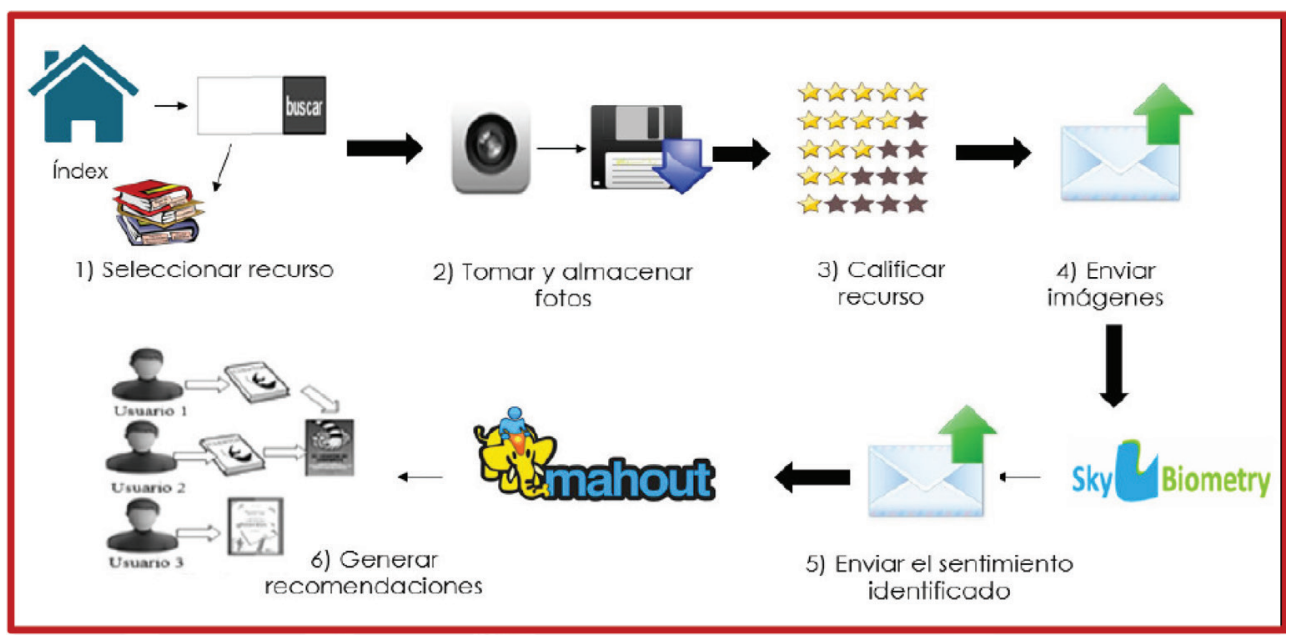

Figura 2 - Proceso de recomendación

1. El usuario accede a la interfaz de EmoRemSys para hacer una búsqueda de un recurso introduciendo un criterio. De los resultados obtenidos selecciona el recurso educativo que se adapte más a lo que necesite

2. En el transcurso de tiempo en el que observa el recurso hasta el cierre de la ventana donde se encuentra, se toman diversas fotos de la cara del usuario con la Webcam y se almacenan para enviarse al módulo de análisis de sentimientos (solicitando permisos para dichas fotos). En el caso de que el usuario no disponga de una Webcam se omite este paso.

3. El usuario realiza la calificación del recurso que acaba de utilizar de acuerdo a su criterio.

4. Las imágenes se envían al módulo de análisis de sentimientos.

5. Se analizan las fotografías del usuario mediante la API de SkyBiometry la cual permite conocer do el estado de ánimo del usuario y se envían los resultados al módulo de recomendaciones (SkyBiometry, s.f.).

6. En el módulo de recomendaciones se procesa la información obtenida por el módulo de análisis de sentimientos y por la calificación que el usuario otorgó al recurso educativo. Mediante el uso de la API de Apache Mahout el filtrado colaborativo ocupara una técnica basada en memoria ya que se emplean métricas de similitud para saber la semejanza entre un conjunto de usuarios y realizar las recomendaciones (Apache, s.f.)

En la Figura 3 se presenta el proceso de obtención del sentimiento. El proceso de adquisición del sentimiento está dividido en dos grandes módulos llamados: Componente obtención y análisis de imagen y Componente obtención y análisis del sentimiento cada módulo a su vez tienen sub módulos que se comunican entre sí para obtener una respuesta, el sentimiento del usuario que interactúa con el sistema. A continuación se describe el flujo del proceso. 
1. El Usuario interactúa con el sistema y selecciona la opción de usar cámara Web directamente al módulo adquisición imagen. Se toma la foto que ahora será llamada imagen.

2. La imagen se envía al módulo validación imagen en el que se analiza si esta imagen tiene un formato valido. Conociendo el formato, la imagen se envía al siguiente módulo.

3. La imagen se almacena en el repositorio

4. Esta imagen (ruta) se almacena directamente en una carpeta propia del usuario y quedará vigente en la carpeta hasta que se reemplace por otra imagen del usuario en futuras sesiones que inicie en el sistema.

5. La ruta de la imagen es enviada al componente obtención y análisis del sentimiento para continuar con el flujo de adquisición del sentimiento del usuario. La ruta de la imagen llega al módulo conjunto de servicios Web como un dato de entrada que será consumido por servicios Web desarrollados en PHP (PHP: Hypertext Preprocessor).

6. Dentro de los servicios Web se realiza la invocación a la API SkyBiometry. SkyBiometry es una herramienta que tiene dentro de sus características, detectar el rostro del usuario en varios ángulos, encontrar la ubicación de los ojos, la nariz, la boca y muchos otros puntos en el rostro plasmado en la foto. SkyBiometry determina el sexo y la edad, comprueba si la persona está sonriendo, tiene los ojos abiertos, los labios sellados o si usa gafas. Todos estos datos en conjunto se analizan para obtener el sentimiento del usuario.

7. La respuesta del sentimiento del usuario se devuelve al servicio Web después de analizarse por la API SkyBiometry.

8. La respuesta obtenida (el sentimiento) se transfiere al módulo analizador de sentimiento para un proceso de decodificación de datos de la respuesta.

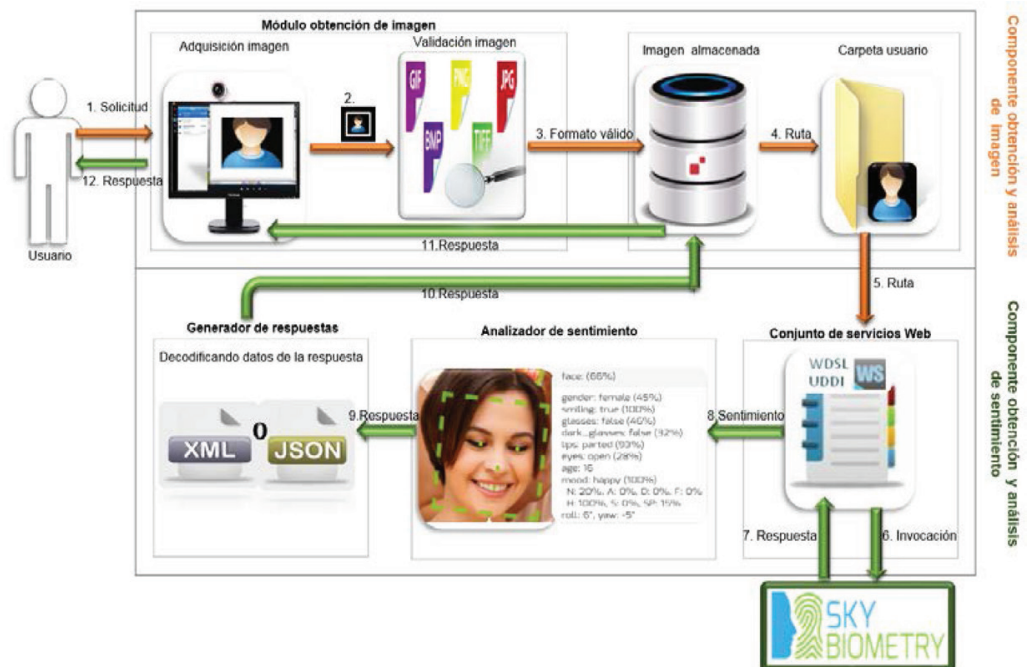

Figura 3 - Proceso de Obtención del Sentimiento 
9. Como resultado de proceso de decodificación de datos de la respuesta se genera una estructura de respuesta dentro del módulo generador de respuesta en formato XML (eXtensible Markup Language) o JSON (JavaScript Object Notation).

10. El módulo generador de respuesta envía al repositorio la respuesta obtenida del sentimiento del usuario el cual es almacenado.

11. Del repositorio se extrae la respuesta para enviarse al módulo de adquisición de imagen como resultado de la solicitud.

12. Finalmente, el usuario conoce su estado, sentimiento y continúa interactuando con el sistema.

\section{Caso de estudio: Generación de recomendaciones de recursos educativos}

En esta sección se presenta un caso de estudio como prueba de concepto de EmoRemSys. El caso de estudio presenta el funcionamiento del sistema de recomendación EmoRemSys mostrando las recomendaciones que se brindaron a un estudiante que ingreso un criterio de búsqueda de la asignatura de matemáticas. En la figura 4 inciso a), se presentan los resultados, una nube de etiquetas con los criterios de búsqueda más regulares de recursos educativos y un sidebar con los recursos más vistos y mejor calificados.

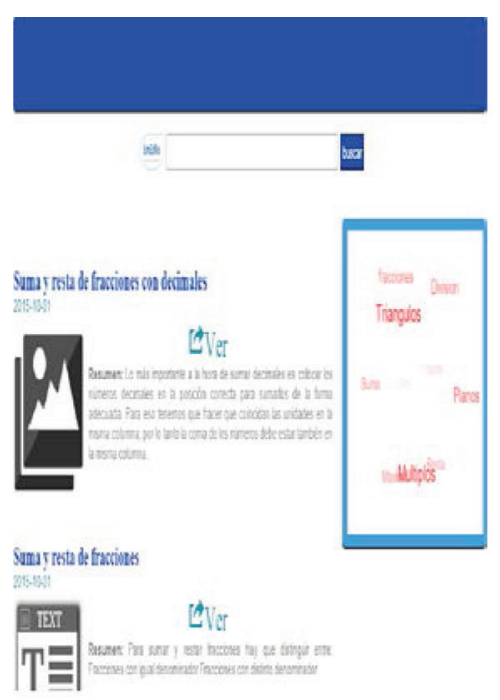

a) Resultados de Búsqueda

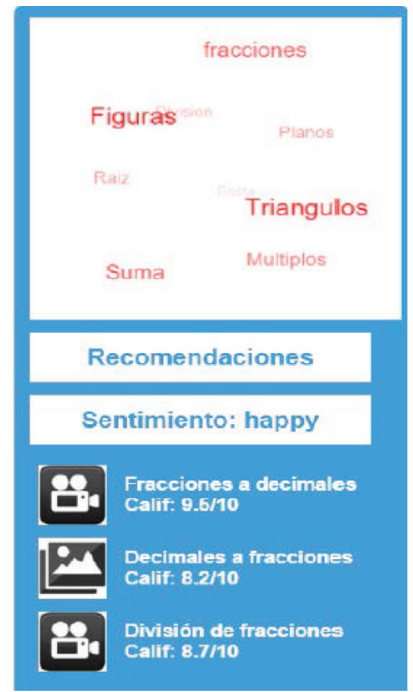

b) Recursos Recomendados

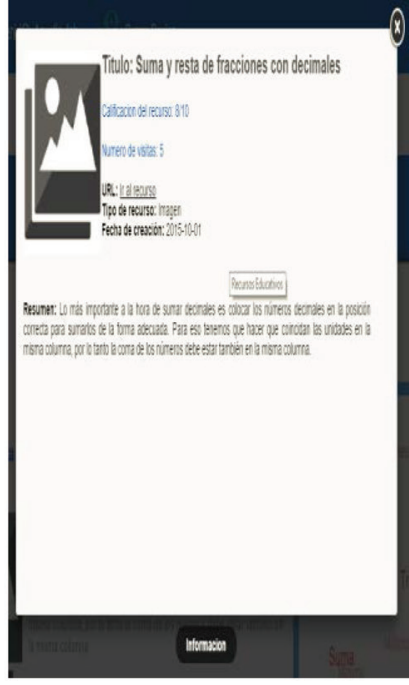

c) Información General de un Recurso

Figura 4 - Interfaces de EmoRemSys

En la figura 4 inciso c), se presenta una descripción general del recurso educativo seleccionado por el usuario incluyendo una url para acceder al contenido del recurso. Después de que el estudiante ingresa al contenido del recurso, se realizan las operaciones 
anteriormente descritas en el proceso de generación de recomendaciones, el estado de ánimo ocupa un factor importante en las recomendaciones de EmoRemSys ya que con éste se conoce de manera clara si el recurso satisface o no al usuario, así como también las emociones en concreto del estudiante las cuales son: feliz, triste, enojado, sorprendido, disgustado, asustado, neutral; éstas son todas las emociones que se identifican con la API de SkyBiometry.

En la figura 5 se presenta un ejemplo grafico de la forma en que trabaja la API de SkyBiometry y la información que presenta, dicha información es recolectada después de procesar las fotos mediante la API haciendo llamadas desde el módulo de análisis sentimental. La información se presenta en formato JSON y esta se recolecta para determinar el sentimiento del usuario y se envía al módulo de recomendaciones.

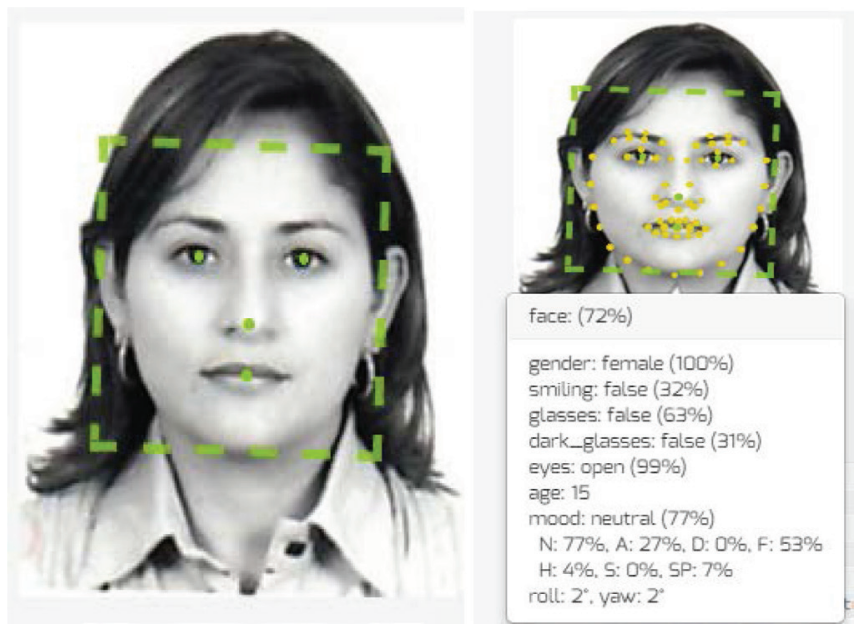

Figura 5 - Ejemplo uso de la API de SkyBiometry

En este caso en concreto se determinó mediante el análisis sentimental que el estudiante presentaba un estado de ánimo neutral por lo cual en la figura 4 inciso b), se presentan tres recomendaciones realizadas al estudiante las cuales son materiales interactivos.

\section{Evaluación}

Para evaluar los algoritmos de filtrado colaborativo que contiene la API de Apache Mahout se realizó el cálculo de "Precisión" y "Recall” (Exhaustividad) del dataset BookCrossing el cual contiene las calificaciones que varios usuarios han dado a diversos libros, este cuenta con 278858 usuarios, 1149780 calificaciones que han dado los usuarios a los libros y 271379 libros. La Precisión se entiende como la probabilidad de que un elemento seleccionado sea relevante o no y el Recall se entiende como la probabilidad de que sea seleccionado un elemento relevante. Las fórmulas para calcular cada una de estas evaluaciones se muestran a continuación: 


$$
\begin{gathered}
\text { Precisión }=\frac{\mid\{\text { recursos relevantes }\} \cap\{\text { recursos recuperados }\} \mid}{\mid\{\text { recursos recuperados }\} \mid} \\
\text { Exhaustividad }=\frac{\mid\{\text { recursos relevantes }\} \cap\{\text { recursos recuperados }\} \mid}{\mid\{\text { recursos relevantes }\} \mid}
\end{gathered}
$$

La evaluación se realizó obteniendo los datos que arrojaban las diferentes métricas que son correlación de Pearson, coseno, distancia Euclidiana, haciendo pruebas de búsqueda de un libro de un usuario en específico, se hicieron las mismas búsquedas con las distintas métricas de similitud y a partir de esto se le realizaron las recomendaciones pertinentes. Con esto se permitió conocer la "Precisión" de las recomendaciones y el "Recall" de cada métrica utilizada. Cabe hacer mención que sin las métricas de similitud entre usuarios las recomendaciones no tendrían sustento. Por esta razón, es de suma importancia tener estas métricas que permitan al sistema conocer las similitudes de los gustos entre los usuarios y así permitir realizar las recomendaciones (Sean Owen, 2011). Los datos ocupados son todos de tipo numéricos y para realizar las evaluaciones correspondientes se ocuparon: el id del usuario, el id del libro y la calificación que el usuario dio al libro. Con esto se observa en la figura 6 los resultados obtenidos de cada métrica de similitud, donde se encontró que la métrica que ofreció mejores resultados fue la correlación de Pearson. La correlación de Pearson es un número entre -1 y 1 que mide la tendencia entre dos series de números, emparejados uno a uno, para moverse juntos. Esto significa si la distancia entre un numero con otro es larga el valor será -1 y entre más cercanos estén, el valor se acercará cada vez más a 1 (Sean Owen, 2011). Los resultados obtenidos muestran que la Correlación de Pearson obtuvo resultados más satisfactorios.

Así también la correlación de Pearson solo considera las asociaciones entre los estudiantes con mismos recursos vistos, en caso contrario no hace los cálculos ya que

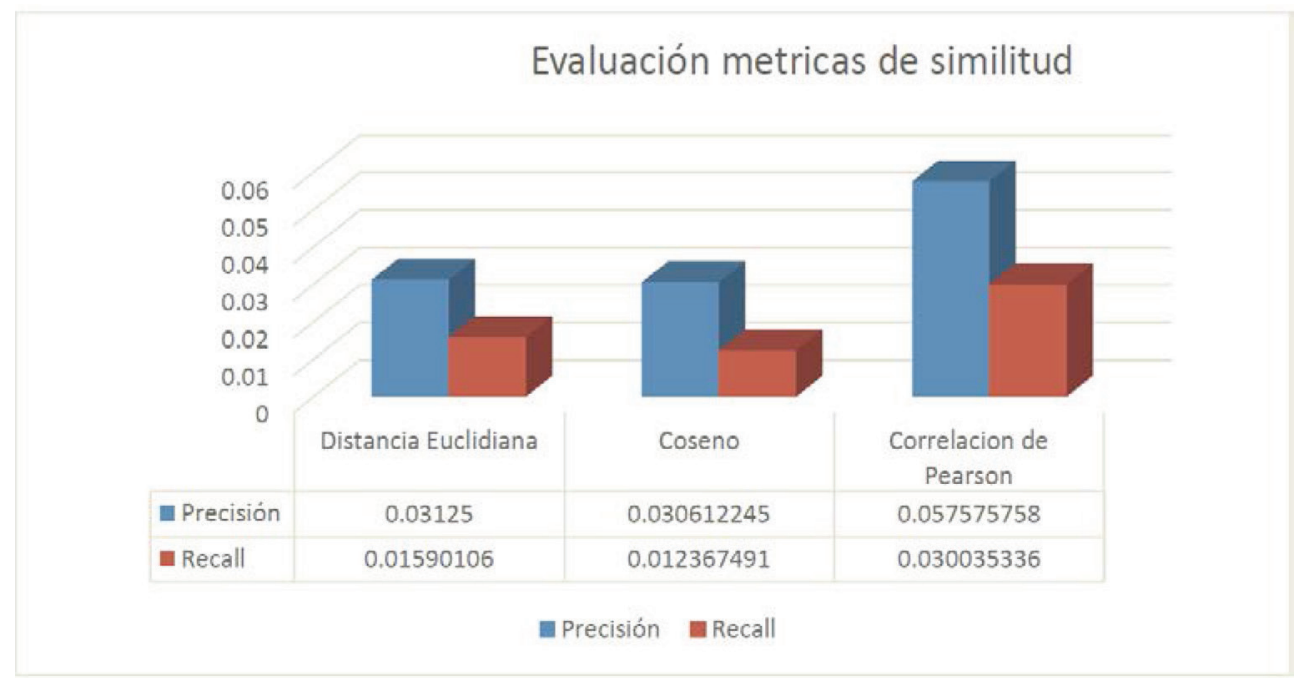

Figura 6 - Evaluaciones de las métricas de similitud. 
si los hiciera a pesar de no tener los mismos recursos vistos se daría una información menos precisa por la falta de datos entre los usuarios lo cual disminuiría la precisión de las recomendaciones. Uno de los inconvenientes de la distancia Euclidiana es que es sensible a las unidades de medida de las variables esto se refiere a que las diferencias entre los valores altos de los datos contribuirán en mucha mayor medida que las diferencias entre los valores de las variables con valores bajos y esto da como consecuencia de ello los cambios en la escala que determinarán también cambios en la distancia entre los estudiantes presentando datos no tan precisos (Introducción al análisis de clusters, s.f.).

En el caso del Coseno no presenta alguna desventaja o algún inconveniente cabe mencionar que la implementación de esta métrica en la API de Apache Mahout presentan resultados bastantes semejantes a la de correlación de Pearson por lo cual en (Sean Owen, 2011) se hace la recomendación de ocupar la métrica de correlación de Pearson.

Adicionalmente, también se realizó una evaluación a la API de SkyBiometry. La API de SkyBiometry permite obtener el sentimientos de una fotografía con un rostro humano, para hacer una evaluación de la precisión de esta API se ocupó una base de datos de rostros llamada Radboud la cual contiene una base de datos de caras, un conjunto de imágenes de 67 modelos (incluidos hombrey mujeres de raza blanca, los niños caucásicos, los niños y las niñas de raza blanca, varones marroquíes así como también holandeses) teniendo 8 expresiones emocionales. El RAFD en una iniciativa del Instituto de Ciencias del Comportamiento del Radboud University Nijmegen, que se encuentra en Nimega (Países Bajos), y se utiliza libremente para la investigación científica no comerciales por los investigadores que trabajan para una universidad acreditada oficialmente (Radboud Faces Database, 2015). La evaluación de dicha API presentó los siguientes resultados que se observan en la figura 7.

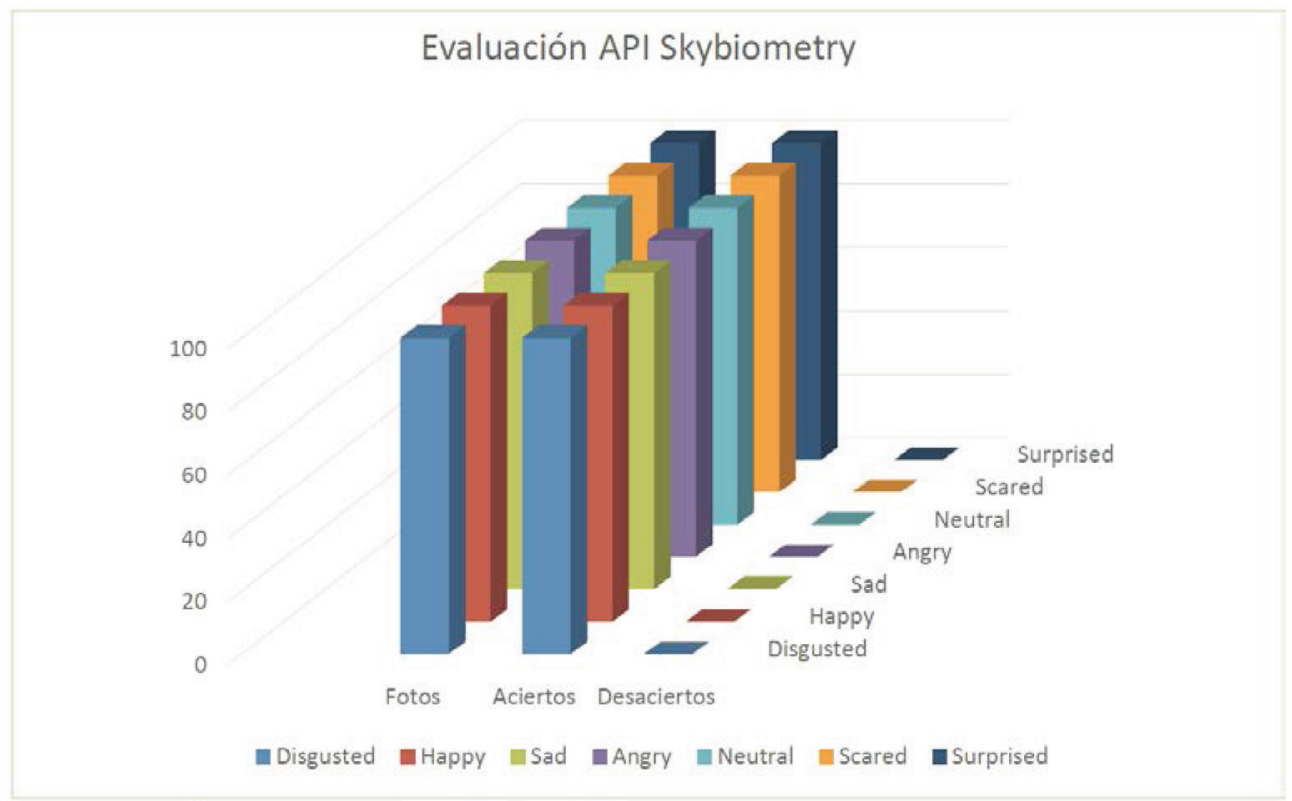

Figura 7 - Evaluación de la API SkyBiometry 
Los resultados que se presentan se obtuvieron haciendo llamadas de 7 en 7 imágenes a la API de SkyBiometry ya que una cantidad mayor presenta un error de tiempo de espera y ya como resultado final de la evaluación se obtuvo que haciendo uso de las imágenes de la base de datos Radboud la API presenta un 100\% de certeza a la hora de predecir el sentimiento de la fotografía tomada, lo cual demostró que ocupar la API de SkyBiometry fue la mejor opción.

\section{Conclusiones y Trabajo a Futuro}

En este trabajo se presentó EmoRemSys el cual aporta una integración de diversas tecnologías para realizar recomendaciones. Se desarrollaron un contjunto de componentes necesarios para el correcto funcionamiento de este sistema, entre los cuáles están: el desarrollo del servició Web encargado de realizar las recomendaciones mediante el filtrado colaborativo con la API de Apache Mahout, los componentes de análisis sentimental en donde se usa la API de SkyBiometry para la detección del sentimiento del estudiante, el analizador de peticiones que sirve como puerta de enlace a la petición que realiza el usuario al momento de ceder o no las funcionalidades de su cámara Web, así como también el uso de diversas tecnologías para complementar el sistema como el uso de CSS3 para los estilos de la página, JQuery para el uso de la cámara Web y las fotografías automáticas del estudiante, Ajax para la funcionalidad de almacenar la fotografía automáticamente en el servidor sin necesidad de salir de la página, el uso de Flash Player para la visualización de los recursos educativos de tipo video en formato.flv para el correcto funcionamiento en los diferentes browser que hay en la actualidad, el uso de Google Docs para la visualización de los recursos educativos que están en formato office, HTML5 para ocupar los formatos en imagen y audio de los recursos.

En la actualidad no hay un sistema con estas características, se comprendió en el desarrollo de este sistema que en la actualidad la búsqueda de la precisión y ahorro de tiempo son muy importantes conociendo la cantidad de información tan extensa que se encuentre en la Web, así como también el uso de la computación afectiva sirve de ayuda para la toma de decisiones. Los resultados finales en el uso de las evaluaciones de "Precisión" y "Recall" mostraron que las recomendaciones del algoritmo de correlación de Pearson son más precisas que los demás algoritmos ya que se ajusta más a datos numéricos.

Como trabajo a futuro se pretende obtener los recursos de diferentes repositorios permitiendo al estudiante escoger de cuántos y cuáles repositorios desea obtener los recursos, los repositorios de recursos educativos son de gran importancia porque con estos permiten tener un mayor volumen de recursos para los distintos niveles de aprendizaje. Además se pretenden desarrollar y tener más filtros en las recomendaciones haciendo uso del filtrado demográfico, del filtrado basado en contenido y otros haciendo así un sistema de recomendación hibrido que tenga mayor precisión, los sistemas de recomendación con filtrado demográfico permiten hacer recomendaciones a partir de los datos obtenidos del usuario como la edad, raza, sexo, lugar de nacimiento entre otros. Lo anterior permitiría generar recomendaciones de los recursos a partir del país de donde se haga la consulta haciendo la recomendación a partir del temario que ocupe cada país para el desarrollo de sus asignaturas. Finalmente, se desarrollará un módulo 
que permita diferenciar el estado de ánimo del estudiante después de la recomendación esto se plantea como mejora una mejora al sistema.

\section{Agradecimientos}

Este trabajo es apoyado por el Consejo Nacional de Ciencia y Tecnología (CONACYT), Tecnológico Nacional de México (TecNM) y la Secretaria de Educación Pública (SEP) a través de PRODEP.

\section{Referencias}

Aguila, J. V. (2010). Distribución de conocimiento y acceso libre a la información con Recursos Educativos Abiertos (REA). La educación.

Apache. (s.f.). Apache Mahout. Recuperado el 18 de Mayo de 2015, de https://mahout. apache.org

Barrón-Estrada, M. L.-C.-O.-P.-A. (2014). Un tutor inteligente, afectivo y configurable para el aprendizaje de números naturales de 3er grado.

Beltrán Páez Germán, G. G. (10 de Julio de 2015). Sistema Recomendador Basado En Filtrado. Obtenido de http://www.enid.unal.edu.co/2012/memorias/fscommand/ tecnologias/59.pdf

Betancur, D. M. (2009). Modelo para la recomendación y recuperación de objetos de aprendizaje en entornos virtuales de enseñanza/aprendizaje Recommendation and retrieval model of learning object in virtual environments for teaching/learning. Revista Avances en Sistemas e Informática, 6(1).

Buder, J. \&. (2012). Learning with personalized recommender systems: A psychological view. Computers in Human Behavior.28 (1), 207-216.

Bustos-Lopez, M. Vázquez-Ramírez, R., Alor-Hermández, G. (2015). An architecture for developing Educational Recommender Systems. 8th Workshop on Intelligent Learning Environments. Research in Computing Science. Instituto Politécnico Nacional. Vol. 106, pp 17-26.

Castillejo, M. M. (2007). El sistema GRADE para la toma de decisiones clínicas y la elaboración de recomendaciones y guías de práctica clínica. Atención primaria, 39(9), 457-460.

Causa, E. \&. (2007). La computación afectiva y el arte interactivo. área Transdepartamental de Artes Multimediales, 52.

Cearreta, I. \&. (s.f.). Aplicación de la Ontología Affinto para el Desarrollo de un Sistema de Conversación Emocional por Texto.

Chia-Cheng, H., Hsin-Chin, C., Kuo-Kuang, H., \& Yueh-Min, H. (2012). A personalized auxiliary material recommendation system based on learning style on Facebook applying an artificial bee colony algorithm. Computers and Mathematics with Applications, 64, 1506-1513. 
Eirinaki, M., Pisal, S., \& Singh, J. (2011). Feature-based opinion mining and ranking. Journal of Computer and System Sciences, 1175-1184.

Herrera-Viedma, E. P. (s.f.). Sistemas de recomendaciones: herramientas para el filtrado de información en Internet.

Hsu, C.-K., Hwang, G.-J., \& Chang, C.-K. (2013). A personalized recommendation-based mobile learning approach to improving the reading performance of EFL students. Computers \& Education, 63, 327-336.

Introducción al análisis de clusters. (s.f.). Recuperado el 27 de 05 de 2015, de http:// www.uv.es/ceaces/multivari/cluster/CLUSTER2.htm

Jorgue Hernández, A. G. (2013). Emociones artificiales usando mecánica cuántica. Computacion afectiva, 2(5), 22-26.

Kaklauskas, A., Zavadskas, E., Stankevic, M. S., Raistenskis, J., Simkevicius, C., Stankevic, T., y otros. (2013). Recommender System to Analyze Student's Academic Performance. Expert Systems with Applications, 40, 6150-6165.

Liu, J. T. (2013). Affective Computing Applications in Distance Education. In 2013 the International Conference on Education Technology and Information System.

Lungu, V. (2011). A framework for developing embodied intelligent agents with affective behavior.

Michael D. Ekstrand, J. T. (15 de Julio de 2015). Collaborative Filtering Recommender Systems. Obtenido de http://files.grouplens.org/papers/FnT\%20CF\%20 Recsys\%20Survey.pdf

Moreno-Cadavid, J., \& Montoya-Gómez, L. F. (2015). Uso de un entorno virtual de aprendizaje ludificado como estrategia didáctica en un curso de pre-cálculo: Estudio de caso en la Universidad Nacional de Colombia. RISTI, 16, 1-16.

Nieto, S. M. (2007). Filtrado colaborativo y sistemas de recomendación. Inteligencia en Redes de Comunicaciones. Madrid.

Sky Biometry. (s.f.). Sky Biometry API. Recuperado el 18 de Mayo de 2015, de Sky Bionetry API. https://skybiometry.com/

Paula N. Medina, O. S.-C. (2013). Desarrollo de un avatar animado con expresión de emociones basicas. Computación Afectiva, 2(5), 6-10.

Pera, M. S., Condie, N., \& Ng, Y.-K. (2011). Personalized Book Recommendations Created by Using Social Media Data. WISE 2010 Workshops, 6724, 390-403.

Poy, R, \& Gonzales-Aguilar, A. (2014). Factores de éxito de los MOOC: algunas consideraciones críticas. RISTI, 105-118.

Radboud Faces Database (2015), [En línea]. Available: http://www.socsci.ru.nl:8180/ RaFD2/RaFD?p=main. [Último acceso: 1511 2015].

Sean Owen, R. A. (2011). Mahout in Action. Manning. 
Tzu-Wei Tsai, H. Y.-S. (2012). An affective computing approach to develop the gamebased adaptive learning material for the elementary students. In Proceedings of the 2012 Joint International. New York.

$\mathrm{Xu}$, Y. G. (2012). Combining social network and semantic concept analysis for personalized academic researcher recommendation. Decision Support Systems. 54 (1), 564-573.

Wang, D., Zhu, S., \& Li, T. (2012). SumView: A Web-based engine for summarizing product reviews. Expert Systems with Application. 40(1), 27-33.

Yang, X. G. (2014). A survey of collaborative filtering based social recommender systems. Computer Communications. 41, 1-10.

Yasmín Hernández, G. A.-F. (2013).Comportamiento afectivo en sistemas de capacitación inteligente para operadores de sistemas electricos. Computación Afectiva, 2(5), $16-18$.

Zhang, J., Wang, Y., \& Vassileva, J. (2013). SocConnect: A personalized social network aggregator and recommender. Information Processing and Management, 49, 721-737. 